\title{
Relation of Pulmonary Venous Fibrillatory Rates to Rates Obtained from the Surface Electrocardiogram in Persistent Atrial Fibrillation
}

\author{
D Husser $^{1}$, M Stridh $^{2}$, L Sornmo $^{2}$, DS Cannom ${ }^{1}$, AK Bhandari ${ }^{1}$, \\ MJ Girsky ${ }^{1}$, SB Olsson ${ }^{3}$, A Bollmann ${ }^{1,3}$ \\ ${ }^{1}$ Departments of Cardiology, Good Samaritan Hospital and \\ Harbor-UCLA Medical Center, Los Angeles, CA, USA \\ ${ }^{2}$ Department of Electroscience and ${ }^{3}$ Department of Cardiology, Lund University, Sweden
}

\begin{abstract}
Dominant atrial rates obtained from the surface electrocardiogram (ECG) during atrial fibrillation $(A F)$ have been shown to reflect right atrial $(R A)$ and coronary sinus $(C S)$ rates. The relation of pulmonary venous $(P V)$ fibrillatory rate to rates obtained from the standard 12lead surface ECG, is, however, not known.

Baseline 10-second 12-lead ECG recordings were made in 25 consecutive patients undergoing PV isolation for AF. After spatiotemporal QRST cancellation, Fourierbased time-frequency analysis was performed and atrial fibrillatory rate was determined. Mean $P V$ fibrillatory rates were slightly higher than $R A$ and $C S$ rates, with no difference between right and left PVs. RA ( $R=.865$, $p<.001), C S(R=.558, p=.025)$ and $P V$ rates $(R=.457$, $p=.033)$ were related with rates obtained from lead V1. There was a positive correlation between $A F$ duration and $R A(R=.545, p<.05), C S(R=.530, p<.05)$ and subsequently $V 1$ rates $(R=.704, p=.007)$, but not between $A F$ duration and $P V$ rates $(R=.087, p=n s)$. This resulted in a negative correlation $(R=-.533, p<.05)$ between $P V-$ $V 1$ rate differences and $A F$ duration.

In conclusion, fibrillatory rates obtained from surface ECG lead V1 reflect RA, CS and to a lesser degree PV rates. As AF progresses, RA, CS and subsequently VI rates increase, while the $P V$ - $V 1$ rate difference decreases indicating a more important role of other atrial parts for AF persistence. Fibrillatory rates from lead VI seem to express overall electrical remodeling in persistent $A F$.
\end{abstract}

\section{Introduction}

During atrial fibrillation (AF), re-excitation occurs during the repolarization phase of the preceding electrical wave, implying that local excitation almost always occurs without any obvious latency beyond the refractory period. The rate of fibrillatory atrial activation exhibits a close inverse correlation with atrial refractoriness [1] and subsequently AF organization expressed as complexity of atrial activation.[2]

Previous studies have shown that the dominant atrial rate can reliably be obtained from the surface ECG. Signal processing techniques of standard ECG leads using spatiotemporal QRST cancellation and Fourier-based spectral analysis of the remainder ECG have been validated by direct comparison with right atrial (RA) and coronary sinus (CS) rates (for a summary see [3]).

More recently, detailed biatrial mapping of $\mathrm{AF}$ has revealed that the pulmonary veins (PV) may exhibit the fastest activation rates and are therefore considered the driving sources of fibrillation in certain cases.[4] The relation of PV fibrillatory rates to rates obtained from the standard 12-lead surface ECG, is, however, not known and was subsequently investigated in this study.

\section{Methods}

\subsection{Patients}

The subjects of this study were 25 consecutive patients with drug-refractory AF who underwent $\mathrm{PV}$ catheter ablation and presented in AF at the begin of the procedure (Table 1). All patients gave written informed consent for the procedure.

Table 1. Characteristics of the study population.

\begin{tabular}{lc}
\hline Age, years & $61 \pm 12$ \\
Male/Female & $20 / 5$ \\
AF duration, months & $2.2 \pm 3.3$ \\
Heart disease * & \\
$\quad$ None (lone AF) & 13 \\
Systemic hypertension & 9 \\
Coronary artery disease & 4 \\
Dilated cardiomyopathy & 3 \\
LAD, mm & $45 \pm 5$ \\
LVEF, \% & $54 \pm 14$ \\
\hline
\end{tabular}

* more than one possible, LAD $=$ left atrial diameter, $\mathrm{LVEF}=$ left ventricular ejection fraction 
Oral anticoagulants were discontinued 4 days and antiarrhythmic drugs 5 plasma half-lives before the procedure.

\subsection{ECG acquisition and signal processing}

PV mapping and ablation were performed according to standard criteria, starting in the superior PVs sequentially or simultaneously. Electrophysiologic atrial studies were performed using catheters introduced percutaneously and positioned in the right atrial wall (dual decapolar catheter), the coronary sinus (decapolar catheter) and the $\mathrm{PVs}$ (quadripolar catheter with an embedded thermistor for mapping and ablation and decapolar circular catheter). Before proceeding with the ablation procedure, 12-lead surface and endocardial electrocardiograms were recorded and stored using a commercial recording system (Bard EP Lab System Duo, Billerica, USA).

Intracardiac rates at high right atrium $(n=21)$, mid coronary sinus $(n=18)$ and pulmonary veins $(n=25)$ were determined using Fourier-based spectral analysis in 10second recordings as described previously.[5,6]
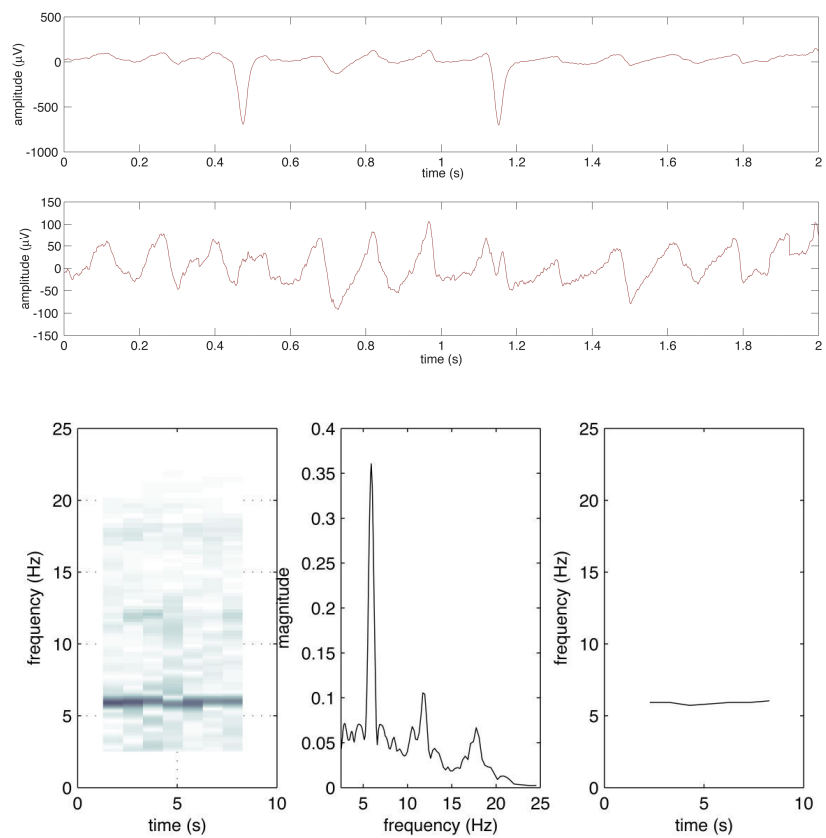

Figure 1. Time-frequency analysis of AF. Two seconds (out of a 10 second recording) of an ECG signal from a patient with AF (upper panel), and the same interval after QRST cancellation (middle panel, amplitude scale is magnified five times). The fibrillatory signal is then subjected to Fourier-based time-frequency analysis. Time-frequency distribution (left box), power frequency spectrum in which mean fibrillatory rate is determined (middle box), frequency trend (right box).
The surface ECG was processed using analysis techniques which have also been described in detail before. Briefly, after analog-to-digital conversion (2000 $\mathrm{Hz}, 12$ bit, $0.05-300 \mathrm{~Hz}$ ) electrograms were stored on optical disk and transferred to a personal computer. After high-pass filtering to remove baseline wander, QRST complexes were subtracted using spatiotemporal QRST cancellation. Since the dominant frequency component of interest is within the $4-9 \mathrm{~Hz}$ range, the resulting fibrillatory baseline signal was downsampled to $50 \mathrm{~Hz}$ and subjected to spectral analysis. The time-frequency distribution of the atrial signal (obtained by short-term Fourier transform) is decomposed such that each spectrum can be modeled as a frequency-shifted and amplitude-scaled version of the spectral profile. This procedure is based on a spectral profile, dynamically updated from previous spectra, which is matched to each new spectrum using weighted least squares estimation. The frequency shift needed to achieve optimal matching then yields a measure of instantaneous fibrillatory rate of a 2.5-second ECG segment (overlapping with one segment each second) and is trended as a function of time.[7]

Frequencies were converted to fibrillatory rates with its unit fibrillations per minute (fpm) as advocated previously $($ rate $=$ frequency $* 60$ ). Mean fibrillatory rate (in fpm) defined as average of instantaneous fibrillatory rates over the two-minute ECG segment was determined. (Figure 1).

\subsection{Statistical analysis}

All continuous variables are presented as mean \pm one standard deviation. Fibrillatory rates obtained from intracardiac and surface recordings were compared using t-test for paired data, correlation analysis, and the BlandAltman method. The possible relation between fibrillatory rates and clinical variables was explored using t-test for unpaired data for continuous data or chi-square test for categoric data and correlation analysis. A p value $<.05$ was considered statistically significant.

\section{Results}

Mean PV fibrillatory rates were higher than RA $(p=.047)$ and CS rates $(p=.05)$, with no difference between right and left PVs. Intracardiac and corresponding rates obtained from leads II $(\mathrm{p}=.017 v s \mathrm{PV}$, $\mathrm{p}=\mathrm{ns} v s \mathrm{RA}$ and $\mathrm{CS}), \mathrm{aVF}(\mathrm{p}=.024 v s \mathrm{PV}, \mathrm{p}=\mathrm{ns} v s \mathrm{RA}$ and $\mathrm{CS})$ and V1 ( $\mathrm{p}=\mathrm{ns}$ vs RA, CS and PV) are depicted in Figure 2.

Right atrial, coronary sinus and pulmonary vein rates exhibited a positive correlation with rates obtained from surface ECG leads, which were most pronounced for lead V1 (Table 2). 


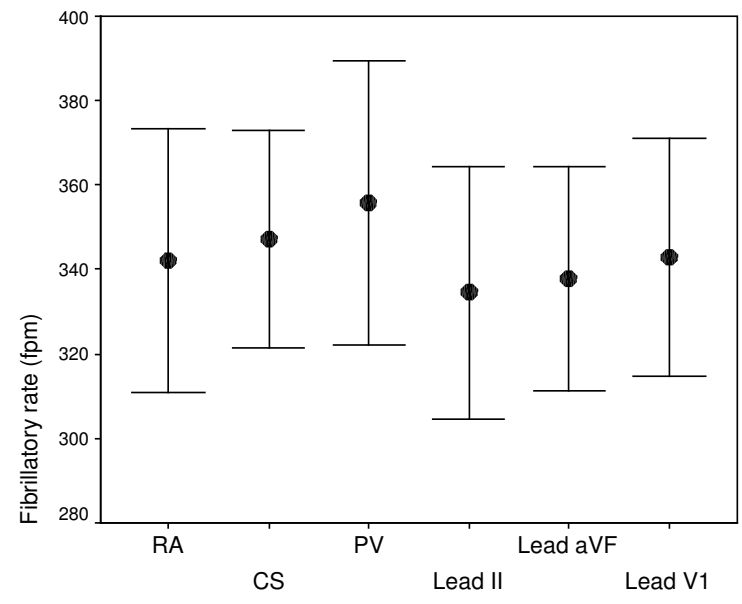

Figure 2. Fibrillatory rates (mean \pm one standard deviation) obtained from right atrial (RA), coronary sinus $(\mathrm{CS})$, and pulmonary vein (PV) electrograms as well as surface ECG leads II, aVF, and V1.

Table 2: Correlations between intracardiac and surface ECG fibrillatory rates.

\begin{tabular}{ccccccc}
\hline Lead & \multicolumn{2}{c}{ RA } & \multicolumn{2}{c}{ CS } & \multicolumn{2}{c}{ PV } \\
\hline & $\mathrm{R}$ & $\mathrm{p}$ & $\mathrm{R}$ & $\mathrm{p}$ & $\mathrm{R}$ & $\mathrm{P}$ \\
\hline $\mathrm{II}$ & .838 & $<.001$ & .349 & .185 & .429 & .046 \\
$\mathrm{aVF}$ & .877 & $<.001$ & .510 & .044 & .391 & .072 \\
$\mathrm{~V} 1$ & .865 & $<.001$ & .558 & .025 & .457 & .033 \\
\hline
\end{tabular}

Figure 3 (Bland-Altman method) illustrates the close agreement of right atrial and coronary sinus fibrillatory rates with rates obtained from simultaneously recorded surface ECG lead V1. Rate differences and variability between ECG and electrograms increased with growing anatomical distance (right atrium - coronary sinus pulmonary veins) to V1 In $59 \% \mathrm{PV}$ rates were faster than V1 rates, while the opposite was observed in the remaining patients.

There was a positive correlation between AF duration and RA, CS and subsequently V1 rates, but not between AF duration and PV rates. This resulted in a negative correlation between $\mathrm{PV}-\mathrm{V} 1$ rate differences and $\mathrm{AF}$ duration. (Table 3).

Table 3: Correlations between fibrillatory rates and AF duration.

\begin{tabular}{lcc}
\hline & $\mathrm{R}$ & $\mathrm{p}$ \\
\hline RA fibrillatory rate & .545 & $<.05$ \\
CS fibrillatory rate & .530 & $<.05$ \\
PV fibrillatory rate & .087 & $\mathrm{~ns}$ \\
V1 fibrillatory rate & .704 & .007 \\
PV - V1 fibrillatory rate difference & -.533 & $<.05$ \\
\hline
\end{tabular}

High Right Atrium vs V1
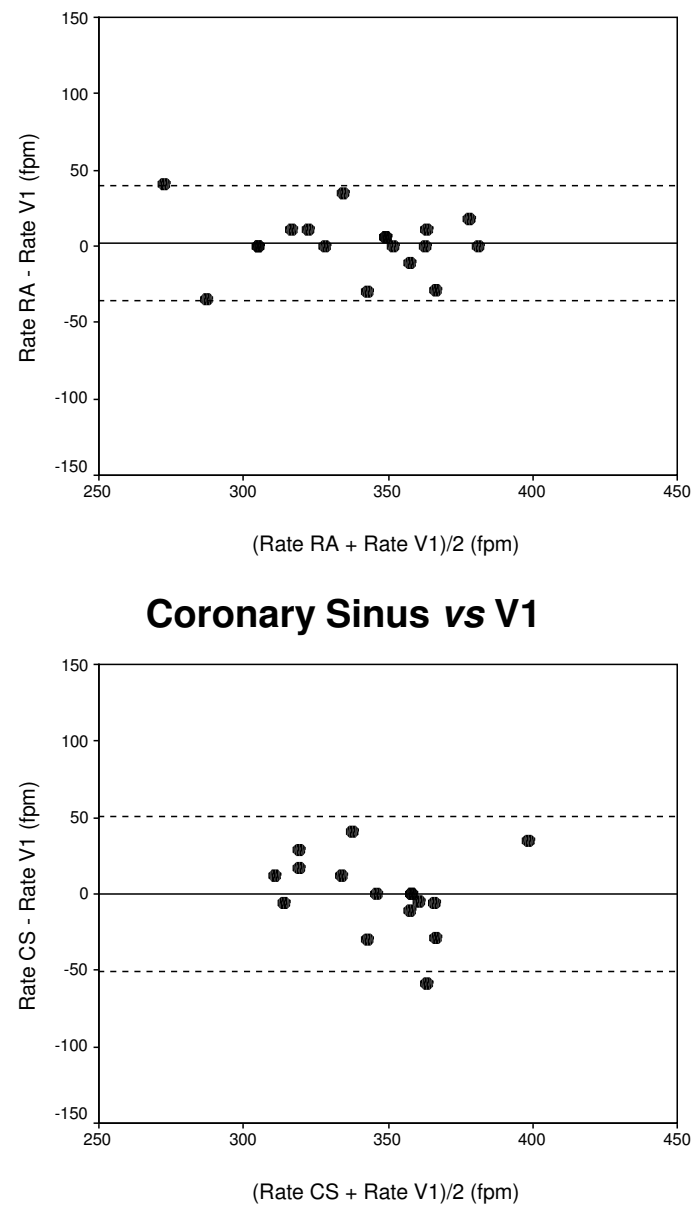

Pulmonary Vein vs V1

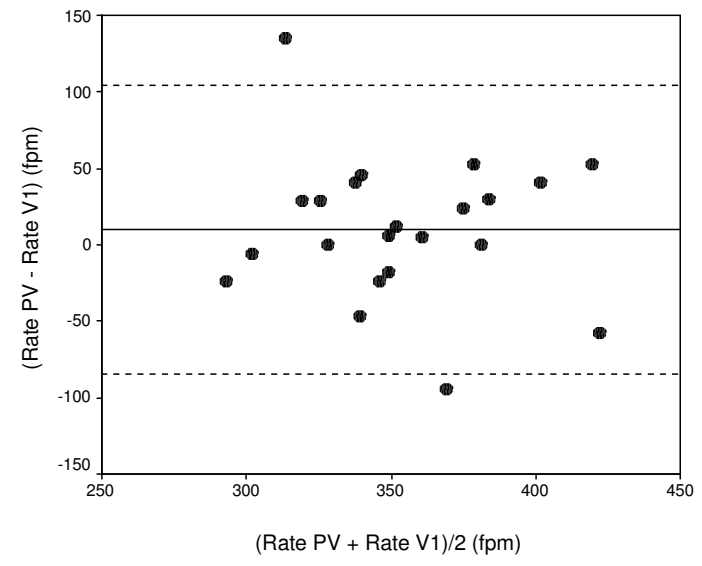

Figure 3. Agreement of fibrillatory rates from the right atrium (top), coronary sinus (middle), pulmonary veins (bottom) with simultaneously recorded fibrillatory rates from surface ECG lead V1 (Bland-Altman method). 


\section{Discussion and conclusions}

This study extends our previous work on the relation of fibrillatory rates obtained from intracardiac and surface ECG recordings by analyzing PV electrograms. As with our previous studies $[5,6]$, there was a close correlation between right atrial and surface electrograms.

Several new findings of this investigation deserve further consideration. The progressive nature of this arrhythmia may be expressed as increase in fibrillatory rates which is in accordance with well-known refractoriness shortening over time.[8] Interestingly, rate increases were restricted to RA, CS and subsequently V1, while PV exhibited no relation with AF duration. These changes resulted in a reduced $\mathrm{PV}-\mathrm{V} 1$ rate difference as $\mathrm{AF}$ was sustained.

These findings taken together fit into the concept that the role of $\mathrm{PV}$ as $\mathrm{AF}$ drivers declines as $\mathrm{AF}$ and subsequently remodeling of other atrial parts progresses. This is supported by the fact that electrical isolation of $\mathrm{PV}$ has been shown to terminate ongoing $\mathrm{AF}$ in $60 \%$, while AF persisted in the other $40 \%$ despite successful $\mathrm{PV}$ isolation. Interestingly, the ratio of our patients with $\mathrm{PV}$ rates $>\mathrm{V} 1$ rates to patients with $\mathrm{PV}$ rates $<\mathrm{V} 1$ rates mirrors exactly the aforementioned $\mathrm{AF}$ termination to $\mathrm{AF}$ persistence ratio of $\mathrm{PV}$ isolation. Therefore, in some patients, AF perpetuation seems not to be dependent on PV activity alone.[9] Moreover, the negative correlation between PV - V1 rate difference and AF duration highlights the possible importance of other atrial parts for $\mathrm{AF}$ sustenance.

V1 fibrillatory rates seem to express overall electrical remodeling, especially in longer-lasting AF. This notion is supported by previous investigations clearly showing the potential clinical value of fibrillatory rate for predicting antiarrhythmic drug responses, atrial defibrillation thresholds and $\mathrm{AF}$ recurrence following cardioversion (for a summary see [3]). If a localized characterization of individual atrial parts is warranted, analysis of an oesophageal lead may better reflect atrial septal and left atrial activity.[6]

\subsection{Conclusions}

Fibrillatory rates obtained from surface ECG lead V1 reflect RA, CS and to a lesser degree PV rates. As AF progresses, RA, CS and subsequently V1 rates increase, while the PV - V1 rate difference decreases indicating a more important role of other atrial parts for $\mathrm{AF}$ persistence. Fibrillatory rates from lead V1 seem to express overall electrical remodeling in persistent $\mathrm{AF}$.

\section{Acknowledgements}

Dr. Bollmann was supported by the Max Kade Foundation, Inc., New York, NY.

The authors wish to thank Charles R. McKay, MD, Division of Cardiology, Harbor-UCLA Medical Center for his continuous support and valuable comments.

\section{References}

[1] Ramdat Misier AR, Opthof T, van Hemel NM, et al. Increased dispersion of "refractoriness" in patients with idiopathic paroxysmal atrial fibrillation. J Am Coll Cardiol. 1992;19:1531-5.

[2] Konings KT, Kirchhof CJ, Smeets JR, et al. High-density mapping of electrically induced atrial fibrillation in humans. Circulation. 1994;89:1665-80.

[3] Husser D, Stridh M, Sornmo L, Olsson SB, Bollmann A. Frequency analysis of atrial fibrillation from the surface electrocardiogram. Indian Pacing Electrophysiol J. 2004;in press.

[4] Wu TJ, Doshi RN, Huang HL, et al. Simultaneous biatrial computerized mapping during permanent atrial fibrillation in patients with organic heart disease. J Cardiovasc Electrophysiol. 2002;13:571-7.

[5] Bollmann A, Kanuru NK, McTeague KK, et al. Frequency analysis of human atrial fibrillation using the surface electrocardiogram and its response to ibutilide. Am J Cardiol. 1998;81:1439-45.

[6] Holm M, Pehrson S, Ingemansson M, et al. Non-invasive assessment of the atrial cycle length during atrial fibrillation in man: introducing, validating and illustrating a new ECG method. Cardiovasc Res. 1998;38:69-81.

[7] Stridh M, Sornmo L, Meurling CJ, Olsson SB. Characterization of atrial fibrillation using the surface ECG: time-dependent spectral properties. IEEE Trans Biomed Eng. 2001;48:19-27.

[8] Wijffels MC, Kirchhof CJ, Dorland R, Allessie MA. Atrial fibrillation begets atrial fibrillation. A study in awake chronically instrumented goats. Circulation. 1995;92:195468.

[9] Oral H, Knight BP, Ozaydin M, et al. Segmental ostial ablation to isolate the pulmonary veins during atrial fibrillation: feasibility and mechanistic insights. Circulation. 2002;106:1256-62.

Address for correspondence

Andreas Bollmann, MD, FESC

Department of Cardiology

Good Samaritan Hospital

1225 Wilshire Blvd.

Los Angeles, CA 90017

USA

Email: andreas.bollmann@medizin.uni-magdeburg.de or andreas.bollmann@kard.lu.se 